Mundo Agrario vol. 18, nº 38, e055, agosto 2017. ISSN 1515-5994

Universidad Nacional de La Plata.

Facultad de Humanidades y Ciencias de la Educación.

Centro de Historia Argentina y Americana

\title{
Visibilización y valorización de la agricultura familiar periurbana. Intervenciones de políticas públicas en el partido de La Matanza
}

\author{
Visibility and recovery of peri-urban family farming. Public policy \\ interventions in La Matanza
}

María Carolina Feito *

* CONICET. Universidad Nacional de la Matanza, Argentina | carofeito@gmail.com

\section{PALABRAS CLAVE}

Agricultura familiar

Desarrollo rural

Intervención social

Periurbano

Buenos Aires

\section{RESUMEN}

Analizamos intervenciones sociales para agricultores familiares periurbanos de La Matanza y articulaciones entre instituciones que implementan políticas públicas para visibilizar estas producciones. Se utilizó metodología cualitativa realizando trabajo de campo etnográfico y relevamiento de información secundaria. Intentamos demostrar que las articulaciones entre distintas instituciones y actores en la implementación de políticas para el sector son aún incipientes y no visibilizan ni valorizan sus potencialidades reales existentes. Descubrimos interfaces $\frac{1}{}$ entre distintos actores: productores, extensionistas e implementadores, así como intervenciones para incorporación de tecnologías apropiadas. Concluimos que las intervenciones operan en una arena de conflicto y disputa en la implementación de políticas y programas de desarrollo.

\section{KEYWORDS}

Family agriculture

Rural development

Social intervention

Periurban

Buenos Aires

\section{ABSTRACT}

The present paper analyzes social interventions adressed to family farmers living in the buffer periurban zone of La Matanza party -Greater Buenos Aires-, focusing on coordination at levels among different institutions that implement public policies oriented to promote and support such agricultural productions. Qualitative methodology was used for ethnographic field work and secondary information survey. Author/s aim/s to demonstrate that the articulations and agreements between institutions and actors are still incipient and do not realize about their real potentialities. The author/s describe/s interfaces between different actors: producers, extension agents and implementers, as well as interventions to incorporate appropriate technologies. The conclusions presented above show that this kind of interventions are being carried out on an conflict scenario and dispute over the implementation of development policies and programs. 


\section{Introducción}

Tenemos que hacernos visibles con nuestras producciones. Ya pasamos el salto de “agricultura con agricultores”, ya vamos por más: “agricultura para alimentar y en manos de los pueblos”, ya estamos politizados (Productor familiar de La Matanza,

octubre 2014)

Analizamos experiencias de intervención social para agricultores familiares periurbanos de La Matanza con el objetivo de describir articulaciones entre distintas instituciones que implementan políticas públicas considerando identificación de ejecución de proyectos conjuntos, relevamiento de capacitaciones hacia productores familiares, posibles superposiciones institucionales de atención.

En un contexto socioeconómico de ajuste general durante los años 90, no hubo respuesta de transformación y adecuación de las instituciones públicas a las problemáticas de este sector del agro, y cuando surgieron alternativas de programas de desarrollo rural (especialmente a partir de la crisis del año 2001), dicha respuesta fue limitada y despareja, resultado de la tensión entre práctica concreta y conflictos de poder y de intereses subyacentes (Manzanal, 2002). Tomamos el caso de la implementación del Programa Prohuerta del Instituto Nacional de Tecnología Agropecuaria (INTA) y del Ministerio de Desarrollo Social de la Nación (MDS), y algunas acciones de intervención realizadas desde el Municipio hacia la población objetivo, de agricultores familiares de las localidades matanceras de Virrey del Pino, González Catán y Lomas del Mirador. Utilizamos metodología cualitativa a partir del trabajo de campo etnográfico con entrevistas a técnicos de programas, productores beneficiarios y observación participante en la labor cotidiana de técnicos del programa Prohuerta, y en reuniones de organizaciones de productores, visitas a las explotaciones y a las agencias de intervención del INTA con injerencia en el partido. Los ejes abordados en las entrevistas fueron: identificar desde qué enfoques se concibe el desarrollo en las políticas de intervención y cómo juegan las políticas públicas (especialmente de municipios y del INTA) en las representaciones sociales de los agricultores familiares. El trabajo de campo fue realizado entre mediados de 2013 y fines de 2015. Además, relevamos información secundaria proveniente de los escasos estudios anteriores sobre el partido (bibliografía de otros autores, informes anuales de proyecto Prohuerta e instituciones que realizan actividades de promoción del sector estudiado, como el Instituto Municipal de Desarrollo IMDES). Utilizamos información complementaria de una encuesta realizada en 2008 por la Subsecretaría de Desarrollo Rural y Agricultura familiar del ex Ministerio de Agricultura Ganadería y Pesca (MAGyP, hoy Agroindustria, MINAGRO -en adelante SAF-) y el Ministerio de Asuntos Agrarios de Buenos Aires a 29 agricultores familiares de Virrey del Pino, citada por Parés (2009).

Descubrimos interfaces entre productores, extensionistas e implementadores del Programa Prohuerta y funcionarios y técnicos del gobierno local que participan en acciones de política pública para el sector agropecuario. Las describimos desde: tipos de relaciones establecidas entre los actores participantes, concreción de acciones articuladas entre distintas instituciones, consideración de aspectos culturales en la implementación del Programa, cómo se define la distribución de recursos y la legitimación de los procesos de intervención de los distintos actores. Además, presentamos una experiencia de intervención social consistente en la incorporación de tecnología apropiada, realizada en Lomas del Mirador por el Instituto de Investigaciones para la Agricultura Familiar -IPAF del INTA-, en la que se analiza la receptividad de los beneficiarios. Destacamos los importantes roles de la agricultura periurbana familiar en el partido analizado. Finalmente, debatimos sobre la orientación de la intervención del Estado frente a la cuestión de los agricultores familiares.

\section{Marco conceptual}

\subsection{Modalidades de intervención social, desarrollo local y políticas públicas}

En interacción con los demás, cada actor social organiza el modo de resolver los problemas de su existencia cotidiana y administra sus relaciones en el marco de redes sociales, realizando una estrategia y movilizando 
recursos dentro de un marco en el que todos los actores administran alguna cuota de poder, aunque sea mínima. La relación de los actores con los agentes externos del desarrollo es un aspecto construido (Long, 1992).

La presencia de intervenciones sociales en forma de políticas, programas y proyectos puede convertirse en un factor significativo al influir en los modos de vida de los actores sociales a partir del aporte de recursos organizacionales, financieros, cognitivos, etc., así como acceso a nuevas redes sociales. A su vez, las acciones y reacciones de los beneficiarios pueden modificar también aquellas intervenciones (Rodríguez Bilella y Tapella, 2008).

Al concebir al Estado como una forma de la práctica social y no como algo fuera de la sociedad, la denominada política estatal o pública se refiere a "un conjunto de acciones y omisiones que manifiestan una determinada modalidad de intervención del Estado en relación con una cuestión que concita la atención, interés o movilización de otros actores de la sociedad civil” (Oszlak, 2007, p. 565). Las tomas de posición del Estado frente a una cuestión siempre asumen un carácter negociado o abiertamente conflictivo.

En Argentina, las intervenciones estatales para productores agropecuarios eran concebidas como políticas agrarias. Actualmente, pueden pensarse como un tipo específico de política social, que constituye un continuum que llega al nivel de desarrollo local:

a) políticas de asistencia: por tratarse de una relación entre un actor que da y otro que recibe;

b) políticas de promoción: entran en juego otros actores (organizaciones de base, entidades intermedias, ONGs, vecinos activos), por lo que se trata de una relación de ida y vuelta entre actor que da y actor que recibe, quien participa en la definición de política y se capacita;

c) políticas de desarrollo productivo: incluyen actores diferentes que provienen del campo privado (empresas, comercios locales), y se articulan fortalezas económicas existentes para desarrollar económicamente la región;

d) nivel de desarrollo local: contiene la mayor articulación entre actores, pues no sólo participa el gobierno municipal sino también el segundo sector (lo privado), y el tercer sector (la sociedad civil) (Arroyo, 2005).

Las formas de intervención externas son entonces necesariamente mediadas y transformadas por los mismos agentes. Diversos dominios sociales se entrecruzan y se construyen mutuamente durante el proceso de implementación de una intervención de desarrollo. Atender a estas “interfaces” de diferentes actores, permite ver arenas de conflicto en las que se negocian prácticas, discursos y sentidos, que, a su vez, modelan dicha intervención (Rodríguez Bilella, 2004).

Respecto de la tecnología en particular, coincidimos con Cáceres (2015) en que "el Estado debería poner en lo más alto de la agenda política la discusión de un modelo agropecuario altamente dependiente del know how tecnológico controlado por empresas multinacionales. El diseño de políticas que fomenten abordajes productivos diferentes (por ej. la agroecología ${ }^{2}$ ) debería ocupar un rol central en la estrategia de desarrollo nacional” (Cáceres, 2015, p. 20). Un elemento clave para el desarrollo de tecnologías exitosas en proyectos de intervención es el trabajo inter y transdisciplinario (Bialakowsky, 2006), y el aporte e inclusión de las comunidades locales en los proyectos (en el diseño, puesta en marcha, desarrollo tecnológico, evaluación, difusión, etc.).

Se requiere otorgar atención prioritaria a niveles municipales o similares, en los que se facilita la interrelación entre estructuras administrativas locales e instituciones de la sociedad civil. Esto se da en contextos de descentralización y desconcentración por los cuales los gobiernos locales tendieron a asumir funciones tradicionalmente desempeñadas por los centrales: fomento de desarrollo económico, mantenimiento de infraestructura, prestación de servicios, protección del medioambiente (Manzanal, 2006).

Una estrategia de desarrollo local debe enfrentar tensiones entre el nivel local y niveles extralocales, entre cooperación y conflicto, entre eficacia y legitimidad. Es así que algunos de los ejes constitutivos del enfoque son: revalorización de la identidad local, articulación público-privada, y participación. El desarrollo local puede 
considerarse como "una compleja combinación de redes en las que se movilizan recursos, se configuran identidades y se consolidan (o redefinen) relaciones de poder” (Craviotti, 2008, p. 57).

Las intervenciones para el desarrollo de un medio rural reconfigurado (sobre todo hacia la última década del siglo pasado, en el contexto de las políticas neoliberales de esa época) se implementaron con base en una definición ampliada de ruralidad, en su doble condición de espacial y social, pero fueron limitadas para intervenir sobre las causas de las situaciones de desigualdad. Por ello, no pudieron cortar el ciclo de reproducción de la misma. Los programas provenientes de políticas públicas tendieron a mostrar una ruralidad más dinámica y compleja, que requiere consideración para modificar las formas de desigualdad y pobreza en el agro rural (Neiman, 2010).

\subsection{El concepto de periurbano y la Agricultura Urbana y Periurbana (AUPU)}

El estudio del periurbano supone el abordaje de un complejo territorial que expresa una situación de interfaz campo-ciudad. De difícil definición conceptual y delimitación, se trata de un territorio de borde, en consolidación, bastante inestable en cuanto a la constitución de redes sociales en el marco de una gran heterogeneidad en los usos del suelo (Barsky, 2005).

La producción agropecuaria allí realizada forma parte del concepto de AUPU, entendida como la producción agropecuaria, su procesamiento y comercialización, realizados en entornos urbanos y periurbanos. Diversos autores destacan sus posibles beneficios: ahorro energético (por las cortas distancias de traslado de productos), provisión de servicios ecosistémicos, estructuración del paisaje, preservación de tejido denso de unidades productivas y áreas verdes, encuadre de procesos de urbanización, mejora de acceso y disponibilidad de productos frescos en áreas urbanas y estructuración de ese territorio. Tiene el potencial de vincularse con modelos de producción sustentables en un sentido integral, como lo es la agroecología, que considera al territorio como un conjunto de relaciones sociales entre diversos actores con un origen común, expresado a través de una identidad, en la cual, al estar referida al "campo", lo agrario cobra una vital importancia.

Sin embargo, la AUPU no es sinónimo en todo caso de producción sustentable, ya que puede coexistir en ella la agricultura familiar, con producción agroindustrial extensiva asociada a un paquete tecnológico que utiliza intensivamente fertilizantes y agroquímicos industriales. También puede generar situaciones conflictivas como la tenencia de la tierra, revalorizada por intereses inmobiliarios residenciales que desplazan la producción agropecuaria.

La producción familiar está presente y es necesaria en la actual estructura económica y social argentina, con sus roles fundamentales en aspectos claves como: ocupación del territorio, producción y provisión de alimentos para el mercado interno, soberanía alimentaria, resguardo y producción de semillas, diversificación de la economía. El agricultor familiar es hoy un actor productivo, no sólo social. Por ello, debe ser sujeto de políticas activas de producción y empleo. Cualquier intervención que busque posicionar a este actor en el sistema agroalimentario debe considerar que:

i) por un lado, la singular estrategia de la Agricultura Familiar -en adelante, AF- (minimizar riesgos, estabilizar rendimientos a largo plazo, diversificar y maximizar retornos) merece un desarrollo tecnológico específico, direccionado y situado, y por tanto, el conocimiento básico que lo sustente debe generarse, desde el punto de vista epistemológico y metodológico en la investigación-acción participativa;

ii) entender que la estrategia de la AF es parte de una relación dialéctica de persistencia y/o resistencia con las economías de enclave de un modelo agroexportador (Feito, 2014).

La AF en Argentina es "una categoría en construcción. Su definición y sus alcances son objeto de múltiples negociaciones en las que intervienen científicos sociales, técnicos, administradores y organizaciones agrarias" (Schiavoni, 2010, p. 43). Si bien no entramos en el intenso debate sobre este concepto, la definimos como un tipo de producción en la cual la unidad doméstica y la unidad productiva están físicamente integradas. La agricultura 
es la principal ocupación y fuente de ingresos del grupo familiar y la familia aporta la fracción predominante de la fuerza de trabajo. La racionalidad del productor familiar es muy diferente a la lógica empresarial, ya que tiene como finalidad la reproducción de las unidades domésticas, distinguidas por el perfil familiar de la unidad, la fuerza del trabajo familiar, la mercantilización parcial de la producción, la indivisibilidad del ingreso familiar, la preferencia por tecnologías intensivas en mano de obra y la pertenencia a un grupo territorial. Es en su interior donde se realiza la transmisión de valores, prácticas y experiencias (Schejtman, 1983; Schiavoni, 2010; Feito, 2014; Obstchatko, Foti y Roman, 2007; FONAF, 2007).

La AF conforma un sector tan heterogéneo como las regiones y producciones a lo largo y ancho del país. De allí la necesidad de "construir un entramado institucional sólido para el diálogo político y la participación organizada" (Lattuada et al, 2012), fundamental para consolidar este sector como un factor del desarrollo rural argentino.

\section{Resultados}

\subsection{El partido de La Matanza como parte del territorio del Área Metropolitana de Buenos Aires (AMBA)}

El municipio de La Matanza es parte de este periurbano que se puede relacionar administrativamente con la región AMBA. En ella viven alrededor de 14,5 millones de habitantes, con una densidad de $1.305 \mathrm{hab} / \mathrm{km} 2$, lo cual representa el 36 \% del total del país y el 74,3 \% de la provincia de Buenos Aires (INDEC, 2010; Ramilo, 2012).

Un fenómeno destacado y estudiado por varios autores (Feito, 2016a; Barsky, 2005; Barsky y Aboitiz, 2011) es el avance de las urbanizaciones privadas, impulsado por un conjunto de actores (inmobiliarias, inversores, gestores y profesionales), dirigidas hacia sectores de alto poder adquisitivo que buscan espacios verdes y menor densidad poblacional (Ratier, 2002). La expansión urbana y los nuevos usos del suelo constituyen una característica general del AMBA, aunque con diferente incidencia en el partido de La Matanza, donde los countries y barrios privados son casi inexistentes. La heterogeneidad en la ocupación del territorio, que permite que cohabiten sectores con alto poder adquisitivo junto con población en situación de pobreza, genera situaciones de complejidad y conflictividad social.

En este nuevo contexto, las actividades agropecuarias sufren modificaciones, en tanto la tendencia hacia usos recreativos y residenciales del suelo impulsa incrementos en la renta de la tierra, pérdida de rentabilidad de los sistemas agropecuarios locales y desplazamiento de las actividades agropecuarias intensivas hacia coronas más alejadas del AMBA (Feito, 2016a; Benencia et al, 2009).

\subsection{Producciones de la AUPU en La Matanza}

En el territorio de La Matanza se encuentran asentamientos urbanos agregados, asentamientos urbanos dispersos, pastizales y áreas de cultivo, las más distantes a áreas urbanas de las cuales se sitúan a unos 8 km (Correa Ayram, 2005, citado en Parés, 2009). La actividad agropecuaria es en general de tiempo parcial, y se combina con actividades urbanas. Considerando la producción alimentaria, el caso de La Matanza tiene relevancia para el estudio por sus características sociales, geográficas, económicas, políticas y de desarrollo que ha adquirido en él la AUPU y la organización de los agricultores (Parés, 2009). Es el único distrito que comprende territorios entre el primer y tercer cordón del AMBA, donde más de 6.000 familias realizan actividades relacionadas con la AUPU, como respuesta en un contexto de desocupación, pobreza y debilidad del Estado.

Este partido es mayormente urbano, excepto la localidad de Virrey del Pino que tiene una importante superficie rural, e integra a la cuenca media del Río Matanza Riachuelo. Tiene 31 kilómetros de largo y algo más de 1.255.288 de habitantes (INDEC, 2001). Las localidades de Virrey del Pino y González Catán son las que más crecieron en el último decenio; concentran 19,5 \% de la población del distrito. En ellas se ubica la mayor parte de las familias que realizan actividades de AUPU. Existe un contexto de desigualdades sociales, desempleo, precarias condiciones laborales, tendencia a la concentración espacial de sectores pobres y debilitamiento de lazos 
comunitarios; muchos de estos rasgos son compartidos con la gran ciudad. Esta configuración social y geográfica es determinante de las posibilidades de desarrollo local. Por ello, la AUPU responde a las necesidades de los actores locales participantes en ella (Parés, 2009).

En muchos lugares del periurbano, el proceso de metropolización tuvo como resultado la ruptura y desaparición de las prácticas agrícolas. En este contexto, La Matanza constituye una excepción. La consolidación de la periurbanización del partido se desarrolló desde principios del siglo XX hasta la década del 40, mediante la división y venta para destino urbano, de superficie destinada anteriormente a actividades agropecuarias. Algunos lotes quedaron para horticultura y otros baldíos. Un indicador del avance de la metrópolis sobre La Matanza, que reforzó su carácter de partido periurbano fue la instalación de establecimientos fabriles en la década del 30 . Esto alteró la estructura socioeconómica y reorganizó el espacio territorial. Así, el proletariado industrial que se fue insertando en el nuevo proceso de desarrollo provenía de las provincias del interior de Argentina, de países europeos y de algunos países limítrofes (Svetlitza de Nemirovsky, 2002).

En 1937 aumentaron los propietarios de parcelas de hasta 5 ha con producción de hortalizas, y se incorporaron antiguos arrendatarios italianos. La pérdida de superficie agraria ante avance de la urbanización obligó a un reacomodamiento de la producción a mediados del siglo pasado. Entre 1947 y 1960 se produce lo que Svetlitza de Nemirovksy et al (1997) denominan modernización del partido: aumenta la población un 308 \% por incidencia de la migración rural urbana y europea, comienza una intensa actividad fabril, se reacomodan sectores productivos, se pierde superficie agraria (cae en un 68 \% el número de parcelas), se acelera la urbanización. En 1993, dada la caída de la producción industrial, aumento de desocupación y pobreza, hasta el año 2000, se produce un proceso de deterioro de precios hortícolas, lo que elimina de la competencia a los propietarios de pequeñas parcelas, los cuales se vieron obligados a vender o arrendar a medianos y grandes productores (Svetlizta de Nemirovsky, 2002).

Según esta autora, desde la primera década de 2000 hasta la actualidad, la estructura social agraria de La Matanza se caracteriza por un desequilibrio en el control de la tierra: un sector de medianos y grandes propietarios hortícolas que domina la producción y comercialización, a través de la compra y arrendamiento de parcelas a pequeños quinteros que las abandonaron (en el período 1993-2000 antes mencionado).

Su cualidad de periurbano explica en parte que las modificaciones en el espacio agropecuario del partido se articulen con el avance de la metrópolis dada la necesidad de nuevos espacios para la actividad industrial. Desde las primeras décadas del siglo XX, se da un proceso de pérdida de superficie agraria, división de las grandes explotaciones, aumento de parcelas pequeñas y crecimiento de la población del partido. Desde los 60, cae el proceso de crecimiento industrial y urbano. El deterioro económico nacional de los 90 produce desequilibrio en la distribución de la tierra, con retroceso de la actividad hortícola por parte de propietarios minifundistas. Esto generó la actual situación de heterogeneidad social e ingreso de nuevos actores al sistema productivo. La dinámica de la estructura social agraria sufre entonces un retroceso hacia un contexto de inestabilidad similar al de las primeras décadas del siglo XX (Svetlizta de Nemirovsky, 2002).

Según el Informe 2008 del programa Prohuerta citado por Parés (2009), la cantidad de explotaciones hortícolas y florícolas del partido era, en 2008, de 32 establecimientos que ocupaban 1.072 ha, de las cuales 917 eran hortícolas, 13 florícolas y 138,5 de viveros. Las unidades de agricultura urbana (AU) (huertas o granjas definidas por el Prohuerta) contabilizaban 3.250 familiares, 54 escolares y 24 comunitarias. En 2012, funcionaban 3.377 huertas, de las cuales 3.303 eran familiares, 67 escolares y 7 comunitarias. Las producciones más importantes desde el punto de vista de los volúmenes producidos eran la horticultura orgánica, la cría de aves y conejos y el cultivo de frutales. Existían otras como el cultivo de aromáticas y medicinales, el reciclado de materia orgánica, la cría de cerdos, ovejas y vacas y el cultivo de forraje para alimentación animal. La mayor cantidad de actividades de agricultura urbana eran huertas familiares realizadas en terrenos privados (5.182 unidades familiares sobre un total de 5.280). Le seguían en importancia las huertas escolares y luego las comunitarias (realizada por grupos de vecinos, asociaciones civiles, asociaciones de productores urbanos, hospitales, cárceles y centros de rehabilitación) que son unas 30, lo que suma un total de 5.280 unidades de agricultura urbana. Aunque la mayor 
parte de unidades de agricultura urbana registradas por el Pro Huerta son huertas, cabe aclarar que alrededor de un $10 \%$ de ellas (520 familias) también realizan actividades de granja, fundamentalmente cría de gallinas ponedoras. La AU se encuentra presente en casi todas las localidades del distrito, sin embargo, casi el $90 \%$ se encuentra en el segundo y tercer cordón. En el primer cordón urbano hay una participación importante en la difusión de la AU de instituciones educativas, centros de jubilados y centros de salud. En el segundo y tercer cordón se da la participación de promotores voluntarios nucleados en grupos comunitarios, iglesias, fundaciones y asociaciones civiles.

Según el relevamiento realizado por Parés en 2009, las actividades productivas comprendidas en lo que denominamos AUPU en La Matanza son: horticultura (huertas familiares, comunitarias, institucionales, emprendimientos productivos), elaboración de abonos y lombricompuesto, cría de animales de granja (principalmente aves y conejos), cultivo de frutales, cultivo de plantas ornamentales para arbolado urbano, y procesamiento de la producción primaria (conservas y alimentos preparados en base a frutas y hortalizas). Este autor estima que las actividades más extendidas en superficie son: agricultura (avance de la soja sobre tierras de cultivos intensivos), ganadería (tambo, bovinos de carne y ovinos), horticultura (realizada a campo) y avicultura (con presencia de empresas integradas).

Por otra parte, según Asiaín (2013), del total de EAPs del AMBA, aproximadamente un 44 \% corresponden al sector de la AF, y ocupan el 19\% de la tierra en producción. Al igual que en el resto del periurbano oeste, en La Matanza conviven explotaciones familiares e intensivas. En el partido, sobre 11.752 ha rurales, la tercera parte (un 34 \%, es decir, alrededor de 4.000 hectáreas) están sembradas con soja. El resto de la producción agropecuaria está preponderantemente en manos de pequeños y medianos productores hortícolas. El avance de la soja produjo un desplazamiento de quintas de frutas y verduras que abastecen al AMBA. La AF en La Matanza tiene $45 \%$ (33 de las 72) explotaciones, y ocupa un 4,14 \% de la superficie total (de poco más de 20 mil ha); la superficie media es de 26,2 has.

En cuanto a comercialización de los excedentes, es frecuente la distribución por parte del mismo productor, en puerta de finca o a domicilio, para completar un circuito corto producto de la baja escala de producción. En algunos casos se agrega valor en origen con el procesamiento de hortalizas, o las conservas. La percepción de estos agricultores es que en todos los casos, independientemente del destino de la producción, esta actividad fortalece la economía familiar (Parés, 2009). La actividad es realizada generalmente por personas no especializadas en agricultura, por nacer en zonas urbanas o por tener actividades anteriores distintas. Este autor distingue dos tipos de actividades para este sector de la AUPU:

i) relacionadas con producción (cultivo huertas, plantas ornamentales, medicinales, frutales, aromáticas, cría de animales de granja y apicultura, producción de insumos -reciclado materia orgánica para abonos y lombricompuesto-, procesamiento de producción primaria -dulces, conservas, vino-, preparación de suelos, siembra, cuidado y cosecha, venta y trueque de productos;

ii) relacionadas con cuestiones sociales y organizativas (capacitación y formación, concientización, promoción territorial y difusión de la AUPU en sus ámbitos de residencia, organización social entre agricultores). En nuestro trabajo de campo encontramos estos dos tipos de actividades. Esto, añade Parés, manifiesta dos estrategias simultáneas: integración vertical de actividades (en el interior de unidades productivas), e integración horizontal (entre agricultores que conforman grupos).

\subsection{Las políticas para las producciones del periurbano bonaerense y su incidencia en las políticas locales matanceras}

Distintos proyectos de actores gubernamentales y no gubernamentales impulsaron actividades relacionadas a la AUPU en el AMBA, comenzaron a reconocer su importancia y a promocionarla desde el retorno de la democracia, a principios de los 80. Estas producciones eran concebidas desde las políticas como respuesta frente a 
la crisis económica y para el combate contra el hambre y la pobreza (Neiman, 2010).

El primer antecedente de apoyo a este sector es del gobierno provincial: la creación de la Dirección de Agricultura Periurbana, en 2007. Luego, en 2009 y 2010 respectivamente, se concretan dos importantes iniciativas a nivel nacional:

i) la apertura de la ya mencionada EEA AMBA del INTA, especializada en AUPU y destinada a prestar asistencia al sector productor de alimentos del área metropolitana, siendo pionera en América latina;

ii) la presentación por parte de la Presidencia de la Nación del Programa Nacional de Agricultura Periurbana de la SAF. Estas iniciativas manifiestan la existencia de políticas públicas en diferentes niveles estatales, que indican decisiones y acciones con mayor especificidad sobre el cinturón productivo rural de Buenos Aires (Barsky y Aboitiz, 2011).

En 2013 se inauguró la Oficina de Desarrollo Local de la EEA AMBA, en la sede de la Región Descentralizada Sur del Municipio en González Catán.

En diciembre de 2014 se sancionó la Ley nacional 27.118 de Reparación histórica de la agricultura familiar para la construcción de una nueva ruralidad en la Argentina, cuya reglamentación está siendo redactada por el Consejo Consultivo de la $\mathrm{AF}^{\underline{3}}$ (Feito, 2016b).

Por otra parte, la Federación de Organizaciones Nucleadas de la Agricultura Familiar —FONAF 4 - nacional realiza acciones articuladas con MINAGRO, a través de la SAF; INTA, a través del IPAF Pampeano, que brinda asesoramiento sobre tecnologías y energías apropiadas, y comercialización y Prohuerta (como vimos, desarrolla acciones entre los productores: entrega de semillas, capacitaciones, seguimiento de los cultivos, asesoramiento organizacional); Municipio, a través de la Secretaría de Producción que realiza capacitaciones; IMDES, ente autárquico creado en 2001 que desarrolla -recientemente junto con la Secretaría de Desarrollo Social local y el Prohuerta- iniciativas para el sector agropecuario matancero. Más tarde se creó el IMDES Rural (Battista et al, 2014), que recibe y administra el dinero de subsidios del ex MAGyP.

La ordenanza municipal 22.658/2012, elaborada según lineamientos de los planes estratégicos 2020 nacional y municipal, trata el desarrollo productivo de La Matanza y fue aprobada casi por unanimidad por el Honorable Concejo Deliberante local. Menciona un “crecimiento sustentable” y el “aumento sistemático de la producción de bienes y servicios, incrementando el valor agregado promedio de la producción en el marco de la preservación del medio ambiente" (Art. 1, inciso 1). El inciso 4 establece: "Fomento de la AF que comprende las actividades agrícolas, ganaderas o pecuarias, forestales, ictícolas, las de producción agroindustrial y artesanal, las tradicionales en recolección y el turismo rural, fortaleciendo la gestión de la unidad productiva y apoyando las inversiones en ella realizadas". El inciso 5 establece: “Implementar políticas de economía solidaria, planificando y gestionando actividades con emprendedores que favorezcan la producción, el crecimiento y el desarrollo económico, con la demanda de trabajo y el desarrollo sostenible, en un marco de equidad y justicia social para la construcción de relaciones de producción, distribución, consumo y financiación basadas en la justicia, cooperación, la reciprocidad y la ayuda mutua”. Se incluyen estos aspectos en una normativa que reconoce el potencial de la actividad agropecuaria en un distrito en el que históricamente fue invisibilizada, por tener un perfil de territorio y una identidad industrialista.

Es innegable el efecto de las políticas nacionales mencionadas en este cambio de visibilidad del sector agropecuario en la política municipal, dado que los primeros subsidios de apoyo y fomento a la AF provinieron del Programa Nacional Periurbano y debían ser ejecutados a través de los municipios, por lo cual la mayoría de estas unidades administrativas debieron comenzar a reconocer la existencia, aportes y potencial de este sector agropecuario en sus territorios (Battista et al, 2014).

En este distrito, existen diversos organismos que promocionan la AUPU, especialmente el INTA, que sostiene acciones desde 1990 (a través del programa Prohuerta que capacita agricultores y promotores, entrega insumos 
como semillas, animales de granja y frutales, e instala centros demostrativos.

Parés (2009) elaboró sobre la base de datos suministrados por Coordinación Pro Huerta AMBA, 2008, una interesante clasificación de gobiernos, instituciones, organizaciones y empresas que apoyan la AUPU en el partido, mediante programas y políticas que implicaron distintas acciones en territorio.

Sin embargo, constatamos en nuestro trabajo de campo que la única intervención social de desarrollo con actividades consolidadas en el distrito (tanto en el tiempo, así como representadas positivamente entre los productores) es el programa Prohuerta, cuyos técnicos recorren las fincas regularmente y organizan reuniones con los productores para detectar problemáticas productivas, de comercialización o sociales, realizan capacitaciones técnicas y en algunas fincas llevan a cabo procesos de transición agroecológica $\underline{5}$.

En las estrategias de intervención estatales estudiadas, tomamos el caso de la implementación del programa Prohuerta en las siguientes asociaciones de productores:

i) Asociación Civil “Los Cirujas”, ubicada en un predio otorgado por el Colegio Lasalle, en González Catán, surgió en 1996 con el apoyo de técnicos de Prohuerta. Actualmente tienen vivero con hidroponía (con subsidio del Ministerio de Asuntos Agrarios provincial) y un centro reproductor de aves (con subsidio del programa Manos a la Obra del MDS);

ii) Asociación “Amaru La Granja Ma Abuakek”, coordinada por un productor referente de la Mesa Local Matanza de la FONAF, tiene una finca en Virrey del Pino, producen verduras, frutas, carne (cerdos, conejos, cobayos), huevos, gallinas, artesanías (hilado, armado de vellón, secado, teñido, colorado, tallado de mates). Está inscripto en Registro Nacional Sanitario de Productores Agropecuarios (RENSPA) y tiene habilitación municipal;

iii) Asociación Virrey del Pino, tienen producción hortícola y participan de la Mesa Local;

iv) Unión de Micro Emprendedores de La Matanza (UNEMA), conformada por microemprendedores, no sólo productores familiares, sino también revendedores de calzado, bazar, textiles, artesanías. Reciben apoyo político y subsidios del MDS, forman parte de la Mesa Matanza FONAF y se agrupan con otras organizaciones extralocales;

v) Mesa Local Matanza de la FONAF, si bien tuvo un rol protagónico en la llegada de políticas para productores del partido -el inicio del Programa Nacional Periurbano-, la mayoría de las organizaciones existía antes de que llegaran sus subsidios, y conformaban esta mesa, que articulaba acciones con distintos organismos nacionales (MINAGRO, a través de la SAF; INTA, a través del IPAF Pampeana y del Programa Prohuerta) y locales (Municipio, mediante Secretaría de Producción y el IMDES).

En todos estos casos relevamos la historia de las organizaciones, las relaciones entre sus miembros y con otras organizaciones, la posibilidad de conformación de organizaciones de segundo y tercer grado, y la concreción de acciones articuladas con otras instituciones.

Encontramos diversos actores, que comprueban las afirmaciones de Parés (2009) acerca de la superposición de acciones de intervención hacia los mismos beneficiarios:

-el gobierno municipal (a través de sus secretarías de Producción, Desarrollo Social, Salud, Educación y Medioambiente)

-el gobierno provincial (a través del Ministerio de Asuntos Agrarios y programas de desarrollo rural)

-el gobierno nacional, a través de instituciones como:

- MAGyP

- INTA

- Instituto Nacional de Tecnología Industrial (INTI), que avala experiencias de transferencia tecnológica 
-Servicio Nacional de Seguridad y Calidad Alimentaria (SENASA) (que controla calidad en las ferias)

- Secretaría de Medio Ambiente (aporta financiamiento)

- MDS (aporta financiamiento)

- universidades con asentamiento o trabajo territorial (por ej.: Universidad Nacional de La Matanza, con investigaciones sobre AF desde el Departamento de Ciencias Económicas y Feria del Productor al Consumidor, desde la Secretaría de Extensión, o Universidad Nacional Gral. Sarmiento, con capacitaciones, investigaciones sobre AF desde el Instituto del Conurbano y la maestría en Economía Social, entrega quincenal de bolsones de verdura a docentes, manejada por el gremio)

-diversas organizaciones de productores.

Respecto de cómo construyen los beneficiarios la relación con agentes externos de los programas (Long, 1992), es importante destacar la fuerte relación que poseen las organizaciones de productores familiares que detectamos, tanto con el INTA (a través de la oficina local mencionada), como más recientemente con la Universidad Nacional de la Matanza, que armó una feria en 2015 que ya lleva varias ediciones.

Estas intervenciones no están exentas de conflictos y tensiones (Manzanal, 2002). En junio del año 2012 se realizó el Primer Plenario de AF de La Matanza, en el cual participaron el Municipio, la SAF y el INTA. "Los representantes de estos organismos no querían cruzarse, al final, no vino ninguno de los tres. Se mostraron producciones genuinas” (productora participante del encuentro).

Las tensiones entre los niveles local y extralocal (Craviotti, 2008) se manifiestan en el manejo de fondos del Programa Nacional Periurbano por parte del Municipio. El gobierno local nombró, a través del IMDES, un representante para los temas rurales de Matanza. La Mesa Local de organizaciones solicita que esos recursos financieros de Nación sean manejados por la Secretaría de Producción local, en lugar del IMDES. Las organizaciones locales tienen una relación un tanto ambigua con el gobierno local porque reconocen la importancia de tener un aliado fuerte e intentan sostener buen trato y confianza con los funcionarios, y realizar acciones comunes en beneficio de los productores, pero tienen reclamos en lo que respecta a la recepción de financiamiento mediante subsidios. "Nosotros tenemos muy buena relación con el Municipio, no podemos ir en contra"; "La plata del Periurbano [Programa Nacional del Periurbano] no coincide con el IMDES, porque las compras las tienen que hacer ellos. Hay que hablar con el director del programa, la plata llega y en el muni te dicen que yo no puedo gastarla así, porque no puedo pagar mano de obra”. (productora hortícola de Virrey del Pino).

Los productores entrevistados son conscientes de la importancia del asociativismo que, según sus testimonios, les permite lograr acciones concretas para mejorar sus producciones y su calidad de vida. Para integrar esta mesa, las organizaciones deben estar en camino de normalización, ya que, si bien ellos no exigen inscripción en el RENAF, es difícil acceder a subsidios y otros beneficios si no están inscriptos y, además, ésta es una condición para ser sujeto de la Ley nacional de AF. La Mesa participa activamente en las actividades de registro y organizó varias ferias de economía social y solidaria, en las que los agricultores familiares exhibían y vendían sus producciones (en distintos espacios públicos -plazas-, y privados - predios de asociaciones y clubes-). "Para esto son las mesas, en esas problemáticas intervenimos todos, relevando la organización y detectando quiénes no tienen la capacidad económica, le armamos el proyecto" (productor coordinador de una organización de Virrey del Pino). "Los productores que nos organizamos somos los familiares, a los grandes productores del partido no les interesa abrir esas puertas.” (productor de González Catán).

También existen tensiones entre las mismas organizaciones, por la captación de recursos externos (Rodríguez Bilella, 2004). "Los proyectos vienen con mirada de técnico: colonizadora. Ellos tienen la solución y saben lo que nosotros necesitamos” (productor de González Catán). "Los recursos públicos se apropian en función de las estrategias políticas: el tractor comunitario actualmente lo maneja un quintero que es presidente de una cooperativa que es nueva y no está bien organizada, a pesar de que tiene en su quinta otros tres tractores propios, y está a 400 metros de otra quinta de N. que no lo usa” (productor de una asociación). 
Del análisis de los resultados de nuestro trabajo de campo -mediante observaciones en reuniones de la Mesa Local y en reuniones de técnicos con productores, entrevistas a líderes, integrantes de organizaciones y técnicos de programas, y a la observación con participación en la feria organizada por la Mesa en el barrio Cristo Rey de Virrey del Pino- surgen distintos elementos de las intervenciones analizadas. Existe cierta tradición de reclamo de las organizaciones de productores a los gobiernos municipales para que planifiquen acciones de apoyo al sector, especialmente en el tema comercialización (solicitan concretamente la creación de una feria municipal). Respecto de las interfaces, crece el afianzamiento de relaciones personales de confianza con los técnicos del INTA. Es escasa la disposición a trabajar en red entre las distintas organizaciones si no es por un objetivo específico común, como la organización de eventos para la venta de productos o capacitaciones puntuales, ya que se muestran competitivas entre sí (tanto por los recursos financieros como por la asistencia técnica). Esto ocurría desde antes de la llegada de los fondos del Programa Periurbano, lo que muestra que las formas de intervención externa son mediadas por los mismos beneficiarios (Long, 1992), así como también que las políticas estatales se insertan en una estructura de negociación y poder (Oszlak, 2007). "Los productores no quisieron llevar mercadería a esa feria porque están luchando desde FONAF hace tres años por armar la Feria de Productores de La Matanza y UNEMA (Unión de Microemprendedores) se separa de FONAF y termina organizando su propia feria. Otras organizaciones apoyan al secretario de AF, en cambio UNEMA viene de la Economía Social y el FONAF son sólo productores agropecuarios, no entran en la reventa, por eso no puede participar de esta feria, son distintas lógicas.” (líder de organización).

En cuanto a las representaciones sociales de los productores acerca de las políticas, coincidimos con Parés (2009) en que ponderan las técnicas de producción ecológicas, como un modo de relacionarse con el medioambiente y respetar los recursos naturales. La actividad agropecuaria es concebida como productora de bienestar y como terapéutica, un modo de tomar contacto con otras personas, compartir y participar. La solidaridad también es un elemento que se repite en los testimonios. Formar parte de la AUPU y cultivar como agricultores familiares permite recuperar saberes y reconocer capacidades propias y de los demás. Esto lo hemos observado en las comunicaciones entre los técnicos del Prohuerta y los productores en sus fincas, en las reuniones de organizaciones y en conversaciones entre productores.

\subsection{La incorporación tecnológica en el sector agropecuario matancero: experiencia de validación de tecnología apropiada en Lomas del Mirador}

Al detectar en los proyectos de intervención locales posibles necesidades de incorporación de tecnología y la receptividad de los productores para acudir al empleo de "tecnologías apropiadas", consideramos que cuando se toma en cuenta la heterogeneidad de la AF, la improcedencia de las recetas o esquemas prefigurados tecnológicos debería tornarse obvia. La generación de tecnologías apropiadas señala la necesidad de desarrollar tecnologías productivas y organizacionales que guarden estrecha relación con las condiciones socioeconómicas y culturales específicas, y con la dotación de recursos con que cuenta este sector particular. Pero no sólo es suficiente que sea “apropiada” en términos de la factibilidad de utilización, sino que también debe ser "apropiable” por los productores, es decir, con posibilidad de transformarse, recrearse y/o adecuarse en función de las necesidades y los cambios que se sucedan en los sistemas productivos y en la comunidad $\underline{6}$.

Cuando hablamos de tecnologías apropiadas debemos definir ciertos requisitos (Prividera et al, 2010): deben ser baratas y accesibles; deben dar respuesta a necesidades básicas y problemas socioeconómicos; deben optimizar la producción total del sistema y los recursos humanos, instrumentales, técnicos y económicos que hagan eficiente y racional el empleo de insumos, para minimizar el uso de insumos externos; deben respetar la cultura e intereses locales; deben permitir que su fabricación, mantenimiento y/o reparación puedan ser hechos por los mismos usuarios o a nivel local, para posibilitar apropiación integral y el control permanente del conjunto del proceso.

En La Matanza se inició, a fines de agosto de 2014, una experiencia de validación de tecnología apropiada para AF mediante la instalación de un gallinero móvil en un centro demostrativo de Prohuerta INTA ubicado en el 
predio del Ex Instituto de Menores Dorrego, en Lomas del Mirador. Durante la misma, realizamos observación participante y entrevistas en profundidad. El microemprendimiento Entre Todos, coordinado por una huertera y su hijo, ya tiene 14 años. En el año 2002 comenzaron a trabajar una huerta como contraprestación del Plan Nacional Jefes y Jefas de Hogar del MDS. Al año siguiente obtuvieron permiso municipal para cultivar con semillas entregadas por Prohuerta y apoyo logístico de la Secretaría de Desarrollo municipal. Iniciaron cuarenta personas, en su mayoría desocupados urbanos, pero se fueron yendo y hubo que conseguir voluntarios. Años después, el Prohuerta instaló un centro demostrativo y, al momento de nuestro trabajo de campo, se estaba incorporando mano de obra del proyecto Argentina Trabaja, del MDS. Producen agroecológicamente -asesorados por un técnicopara consumo propio y venta en el lugar o en los alrededores urbanos. Allí tienen cincuenta gallinas y una huerta con distintas variedades de verduras y plantas aromáticas.

La tecnología a la que nos referimos es un gallinero móvil que fue diseñado por alumnos de la carrera de Diseño de la Facultad de Arquitectura, Diseño y Urbanismo de la Universidad de Buenos Aires (FADUBA), conjuntamente con técnicos del IPAF Pampeana INTA. Esta tecnología es modular, con ruedas y patas altas y forma hexagonal para que no se aplasten las gallinas entre ellas; se arma en sólo veinte minutos y se puede construir con materiales sencillos (dos palletes, serrucho y clavos). Los responsables de la huerta-granja se mostraron entusiasmados con esta nueva tecnología que, dicen, les soluciona problemas cotidianos de la cría de gallinas. La idea de validar esta tecnología antes de producirla en serie a través de empresas o cooperativas asociadas a la Cámara de Maquinaria para la AF y comercializarla es que los productores intervengan sobre el prototipo. Éstos ya observaron errores de diseño (con la distancia entre barras se escaparán los pollitos chicos; no sirve para pollos bebés por el piso abierto; servirá sólo para pastoreo de gallinas). La confianza entre técnicos y el personal de la huerta permitió la selección de esta finca y la recepción abierta de los alumnos de la FADUBA. La productora expresó que se sentía un agente más de la aplicación de esta tecnología, ya que los técnicos dejaron en sus manos la valoración y posibles correcciones. Esto muestra la importancia de la participación consensuada y articulada de productores y técnicos en los procesos de aplicación y validación de tecnologías en el marco de intervenciones de desarrollo.

\section{Análisis de las intervenciones sociales en La Matanza}

En nuestro análisis de la implementación de acciones de intervención por parte de distintos organismos en algunas localidades de La Matanza encontramos que diferentes organismos públicos emiten avales, apoyo y subsidios, según el tipo de organización beneficiaria de que se trate: aquellas de agricultores familiares reciben apoyo de la SAF del ex MAGyP (actual MINAGRO), en tanto, las de microemprendedores, que incluyen otro tipo de actor social no productor agropecuario, reciben apoyo del MDS. Las organizaciones se identifican en sus narrativas y discursos fuertemente con el organismo público que les da apoyo y esto depende del tipo de relación que tiene cada organismo público entre sí. Algunas organizaciones buscan articular acciones para recibir financiamiento externo: “Cuidemos dinero del Estado que es nuestro dinero", en tanto otras prefieren una estrategia más autogestionaria: "Nosotros para organizarnos no necesitamos la plata del Estado, nosotros podemos organizarnos hasta en las sombras (...) no queremos la plata del Estado, nos podemos arreglar solos (...) No venimos a buscar plata, venimos a construir política pública, la política no es partidaria, es pública. Ése es el sentido de la FONAF” (productor miembro de la Mesa Local Matanza FONAF).

El conflicto de políticas puede atribuirse a la presencia en el aparato estatal de organismos con diverso grado de autonomía que entran en tensiones cuando debe definirse la posición del Estado frente a una cuestión social. La ambigüedad no es intrínseca a la toma de posición del Estado, sino producto del enfrentamiento entre algunas de sus unidades (sobre el modo de definir la cuestión o el modo de resolverla), que obedecen a intereses organizacionales y clientelísticos contradictorios (Oszlak, 2007).

En tanto, el Municipio no llega a considerar (ni en los discursos de los funcionarios, ni en las acciones de intervención) a los productores analizados como actores productivos de la economía local. "Los productores son 
pocos, no hace falta una gran planificación para atenderlos" (funcionario municipal). "Los municipios no tienen reconocido el periurbano. Hay un conflicto entre dos provincias distintas: el AMBA y el interior de Buenos Aires" (productor participante de la Mesa Local). "Desde IMDES, no saben dónde hay productores, no saben ni llegar a las fincas, se pierden” (líder de organización).

Respecto de los distintos dominios sociales que habíamos mencionado, que se entrecruzan durante la implementación de una intervención de desarrollo, distinguimos aquí sujetos locales que acumulan capacidades de gestión colectiva (los productores), así como sujetos que representan la intervención de instituciones externas como INTA y gobierno local (los técnicos del programa y los funcionarios gubernamentales). Los líderes de las organizaciones de productores locales administran una cuota mayor de poder debido a su mejor acceso a contactos de instituciones gubernamentales (SAF, Municipio, INTA, universidades que trabajan en territorio). La concreción de acciones específicas como el armado de ferias de productos con recursos de organismos públicos participantes, que generan a su vez redes para administrar dichos recursos (en las que participan miembros de las organizaciones, funcionarios del IMDES del gobierno local, técnicos del INTA, técnicos de SAF MINAGRO), muestra la capacidad de agencia de estos líderes (Long, 1992).

Determinadas acciones descubren la negociación con modelos de interacción de las instituciones involucradas: convocatorias a reuniones, llamados telefónicos, traslado de agricultores a las sedes de las instituciones locales en la localidad de San Justo, o de las extralocales en la Ciudad de Buenos Aires, visitas de los funcionarios y técnicos a las fincas.

Las áreas de incertidumbre "naturalmente provocadas" por los problemas y necesidades de la población beneficiaria (falta de recursos financieros, problemas de acceso a crédito) se intersecan con las áreas de incertidumbre "artificiales o inducidas", consecuencia intencional de la intervención externa (Rodríguez Bilella, 2004) - confusión en fechas de otorgamiento de subsidios, falta de claridad por parte de técnicos de INTA y funcionarios municipales para justificar por qué no llega el dinero-. A los actores estatales relevados, en general, les interesa favorecer a los agricultores en la medida de conseguir aportes a su capital político como funcionarios. En tanto, la población beneficiaria disputa el capital económico necesario para desarrollar sus actividades, por eso este campo o arena es construido a partir del conflicto y la negociación permanentes.

\section{Consideraciones finales}

La tercera parte del territorio del municipio de La Matanza es rural. Las actividades allí desarrolladas pueden ser encuadradas dentro del concepto de AUPU por su estrecha relación con las áreas urbanas circundantes, así como también pueden ser catalogadas como AF, ya que son llevadas a cabo tanto por organizaciones sociales, como por familias productoras. La implementación del Programa Nacional de Agricultura Periurbana de la SAF ex MAGyP facilitó la inclusión del sector de la AF en la agenda política local y generó mejores condiciones para la visibilización del sector agropecuario en general y el de la AF en particular.

Sin embargo, el desarrollo es concebido desde un enfoque tecnicista y economicista, más que desde uno integral que considere aprovechar las potencialidades existentes. Esto se refleja en la escasa vinculación del sector con el resto de la economía del partido, a la vez que es incipiente el reconocimiento por parte de las políticas públicas locales del carácter económico de estas actividades, particularmente de la AF.

Por ello, el fortalecimiento de lazos asociativos entre los productores y la consolidación de vínculos con los distintos niveles de gobierno, así como con el programa Prohuerta y las otras instituciones y actores participantes, pueden mejorar el desempeño de las producciones de la AF y aportar a un proceso de transición agroecológica.

Diferentes actores en el ámbito de la práctica en el terreno, por un lado, y en el ámbito administrativo por otro, representaron y actuaron de diferente manera, en relación a la implementación del programa: mientras que los productores representaron su participación en el mismo como forma de acceso a recursos, los técnicos extensionistas vieron la posibilidad de mejorar su inserción en la estructura institucional. 
Los productores analizados se apropiaron del discurso de reconversión productiva del programa Prohuerta para alcanzar las ventajas que ofrece. De distintas maneras, negocian el alcance de recursos que de no formar parte del programa no conseguirían (provisión de semillas, asesoramiento en fortalecimiento organizacional y presentación de los papeles para formalizar la asociación).

Refutamos la visión transferencista de la extensión, que presenta a los técnicos como implementadores mecánicos de un esquema planificado, ya que en La Matanza no se presentan ni como agentes que han internalizado la ideología de intervención del programa, ni como sujetos pragmáticos que lidian con las dificultades de la implementación.

A partir de los planteos de Arroyo (2005), podemos decir que las políticas implementadas tanto por INTA como por el Municipio son de promoción, ya que se produce un ida y vuelta entre el que da y el que recibe, que fortalece la relación Estado-sociedad civil pero sólo en el nivel de las políticas sociales, sin vinculación con lo económico.

Sigue pendiente la inclusión del sector de la AF matancera en el marco de una estrategia sostenida de desarrollo orientada a la inclusión social, la sustentabilidad económica de la producción local y el respeto y aprovechamiento sustentable de los recursos naturales. El fortalecimiento de lazos asociativos entre los productores y la consolidación de vínculos con distintos niveles gubernamentales pueden mejorar el desempeño de las producciones de la AF y aportar a un proceso de desarrollo local. El Prohuerta, en particular, promueve el fortalecimiento del asociativismo, el acceso a nuevas redes sociales y el desarrollo de capacidades de gestión.

Pero no sólo hay que analizar las instituciones implementadoras sino también las acciones y mundos de todos los actores (Rodríguez Bilella, 2004). Las intervenciones sociales pueden modificar los modos de vida de los actores participantes mediante nuevas relaciones sociales, apoyo de recursos humanos, técnicos y económicos, fortalecimiento organizacional, contactos intra e inter organizaciones, conocimiento de nuevas formas de gestión de recursos.

Los productores familiares periurbanos estudiados mediatizan a través de las organizaciones que integran los flujos externos provenientes del Estado, y así encuentran soluciones concretas a sus problemas; los que hacen agricultura agroecológica legitiman la reconversión productiva a través del aval de los técnicos del programa, en tanto la mayoría consigue financiamiento y/o apoyo logístico para realizar ferias locales de comercialización de sus productos, asesoramiento organizacional y capacitaciones. En la intervención analizada del programa Prohuerta en el Centro Demostrativo "Entre todos", el INTA utilizó una estrategia de reconversión hacia la agroecología.

La instalación de prototipos para que sean validados en fincas de productores es parte de un proceso de evaluación del desempeño de las tecnologías para comprobar que realmente son "apropiadas” a las necesidades reales de los agricultores. Por otra parte, la articulación de un organismo nacional como el IPAF con una universidad nacional como la UBA permite lograr desarrollos complejos con enfoque integral.

En definitiva, la perspectiva teórica y metodológica del enfoque orientado al actor permite entender que los programas son "construidos" y moldeados por diferentes interacciones de múltiples actores. Con la utilización del análisis de interfases se comprende la forma en que los intereses, metas, percepciones y estrategias de diversos actores vinculados al programa resultan continuamente readaptados en función de las múltiples interacciones entre los agentes locales y extralocales. Sin embargo, las complejas interacciones entre los "proyectos" y prácticas de los actores y sus resultados esperados e imprevistos crean tanto marcos habilitantes como limitantes para la acción social. Al focalizarse en la interacción de las relaciones internas y externas este enfoque procura brindar una perspectiva más dinámica sobre las intervenciones de desarrollo y los procesos de implementación de políticas (Long, 1992).

Avanzar en la transformación de aspectos estructurales y productivos (del sector en general y de los agricultores familiares analizados en particular) requeriría la implementación de políticas que modifiquen las actuales condiciones vinculadas con el uso y acceso a la tierra, el capital y las formas y organización social del trabajo. 
De acuerdo a lo sugerido por el Foro de Universidades (2011), coincidimos en que esto podría trabajarse a distintos niveles en el partido analizado:

i) jurídico: concreción de la reglamentación de la Ley nacional 27.118 y nuevas ordenanzas municipales que apoyen al sector (sobre todo respecto del acceso a la tierra y creación de nuevos programas de desarrollo, normativas de comercialización y sanidad que permitan abrir un camino hacia la aplicación de buenas prácticas agropecuarias, así como mejorar la trazabilidad en la cadena de distribución alimentaria y conseguir canales cortos que beneficien a los agricultores familiares);

ii) laboral: garantizar sistemas de trabajo protegidos y formalizados y la erradicación de trabajo indigno (mediante controles del trabajo en las explotaciones);

iii) tecnológico: generación participativa e interinstitucional de tecnologías apropiadas y apropiables (replicar en otros lugares del partido la experiencia realizada por el IPAF y conseguir que el Municipio, desde sus distintas Secretarías, apoye estas iniciativas);

iv) acceso diferencial al crédito para la AF, reforma tributaria que desgrave alimentos (generación de nuevas líneas de crédito desde el Municipio y difusión de información sobre líneas ya existentes, vinculación con la actividad privada que permita obtención de créditos a baja tasa de retorno, con organizaciones tales como la Cámara de Fabricantes de Maquinarias para la Agricultura Familiar - CAMAF-);

v) comercial: promover formas de comercialización sustentables, de canales cortos y sin intermediarios a mercados de proximidad conservando agrobiodiversidad con inclusión social y arraigo de los productores y reestableciendo el lazo productor-consumidor; difusión al consumidor de las ventajas de los productos de la AF; fortalecimiento de la soberanía alimentaria (promover y sostener desde el Municipio ferias itinerantes en espacios públicos que se sumen a las ya existentes en el predio del INTA AMBA y en el de la Universidad Nacional de La Matanza);

vi) organizativo: promover asociativismo y fortalecer las organizaciones locales ya existentes (mediante convenios entre organizaciones para lograr acciones comunes, gestión de microemprendimientos asociativos);

vii) político: organizar gestión asociada entre productores y Municipio tomando a los primeros como sujetos políticos en el marco de una cultura participativa; desplazamiento de los clientelismos y mayor autonomía respecto de los poderes constituidos; promoción de mesas multiactorales o consejos asesores locales conformados por una multiplicidad de actores involucrados.

En suma, integración, eficiencia y sustentabilidad son claves para el futuro de la AF.

Los elementos de eficiencia de este sector, en contraste con la explotación capitalista, están dados por la mano de obra familiar, que estructura tanto procesos sociales como productivos de la explotación, y el grado de mercantilización, que es una ventaja competitiva por el uso de relaciones sociales no mercantilizadas.

En cuanto a sustentabilidad, es necesario promover la transición agroecológica de estos agricultores matanceros, con la creación de programas y proyectos que impulsen este modo de producción que busca maximizar productividad minimizando el uso de insumos externos, y que respeta y pone en valor la cultura local protegiendo el ambiente y pagando salarios justos.

La trayectoria histórica de la AF demuestra la posibilidad de su activación política si se lograra mayor visibilidad de sus aportes económicos y de su inserción territorial (Neiman, 2010). La construcción de nuevas intervenciones o ampliación de las ya existentes se relaciona con el desarrollo y fortalecimiento de modalidades organizativas de integración con otros sectores, que articulen demandas gremiales con acciones económicas, para mejorar las condiciones productivas y de vida de estos agricultores. 


\section{Notas}

1 Las interfaces definen áreas de conocimiento e interacción que entrecruzan las perspectivas de una gran diversidad de actores (estatales, no gubernamentales, la población beneficiaria, los proveedores del crédito, tecnologías, maquinarias, herramientas, insumos, etc.). Es decir, se trata de un campo socialmente construido a partir del conflicto y la negociación en el que se define la distribución de recursos y la legitimación de los procesos de intervención de los distintos actores.

2 Miguel Altieri (1983: Agroecología, Bases Científicas de la Agricultura Alternativa. División de Control Biológico. Universidad de California, Berkeley) define a la agroecología como una ciencia que estudia los principios sobre los cuales se debe basar el diseño de una agricultura sustentable económica, social y ambientalmente: una agricultura ambientalmente sana, diversificada y que rompa el monocultivo como estrategia productiva, y elimine así la dependencia de insumos agrotóxicos externos que son caros y ecológicamente peligrosos, para buscar viabilidad económica y justicia social. Debe complementarse con políticas agrarias de seguridad alimentaria, conservación de recursos naturales y eliminación de pobreza rural.

$\underline{3}$ El Consejo Consultivo de la Agricultura Familiar, Campesina e Indígena es un cuerpo integrado por 13 organizaciones campesinas e indígenas de nivel nacional, que junto a representantes del Ministerio de Agroindustria, INTA y SENASA y el recientemente incorporado Foro de Universidades Nacionales para la AF (del cual participa la autora, representando en el Foro Región Pampeana a la Universidad Nacional de La Matanza); asesora a la SAF del MINAGRO sobre aplicación de políticas públicas para el sector.

4 Ex Foro Nacional de la Agricultura Familiar originado en diciembre de 2004 a partir de la Comisión de Agricultura Familiar generada en el ámbito de la Cancillería por iniciativa de la Federación Agraria Argentina (FAA) y como consecuencia de las propuestas surgidas del "Congreso Nacional y Latinoamericano sobre Uso y Tenencia de la Tierra”, del que habían participado delegados de más de 150 organizaciones de agricultores familiares y de comunidades indígenas. Se convirtió en 2014 en Federación y suma más de 900 organizaciones que asocian a unas 180 mil familias de productores en todo el país.

$\underline{5}$ Avanzar en un proceso de transición hacia sistemas agroecológicos de producción requiere no sólo un conjunto de tecnologías apropiadas, sino conocer argumentos y variables que influyen en la toma de decisiones de los productores, las estrategias colectivas de organización que fortalezcan las negociaciones y las políticas públicas y redes institucionales que sostengan la sustentabilidad de estas experiencias. Ver Marasas, M. (2012), "El camino de la transición agroecológica”, ed. INTA, Buenos Aires.

$\underline{6}$ La agroecología es un paradigma alternativo que centra su accionar en la problemática de la pobreza y de la crisis ambiental, a las cuales reconoce como síntomas producto de decisiones políticas centradas exclusivamente en la acumulación de la riqueza y que favorecen la exclusión de la agricultura familiar. La agroecología busca generar conocimientos que promuevan el desarrollo sustentable (Alteri, 1983, Agroecología, Bases Científicas de la Agricultura Alternativa. División de Control Biológico. Universidad de California, Berkeley).

\section{Bibliografía}

Arroyo, D. (2005). Modelos de gestión municipal y niveles de planificación en Argentina. Posgrado en Desarrollo Local y Economía Social 2005. Buenos Aires: FLACSO.

Asiaín, A. (2013). González Catán y Virrey del Pino: La soja matancera hace su aparición. Diario NCO. Recuperado de http://noticiasconobjetividad.wordpress.com/2013/03/27/gonzalez-catan-y-virrey-del-pino-la-sojamatancera-hace-su-aparicion/

Barsky, A (2005). El periurbano productivo, un espacio en constante transformación. Introducción al estado del debate, con referencias al caso de Buenos Aires. Scripta Nova, revista electrónica de geografía y ciencias sociales, 9(194), (36). Disponible en: http://www.ub.edu/geocrit/sn/sn-194-36.htm (entrada 14/08/2017) 
Barsky, A. y Aboitiz, P. (2011). La agricultura periurbana en la agenda pública. Implementación de políticas municipales, provinciales y nacionales para el sostenimiento del cinturón verde en los bordes de la Región Metropolitana de Buenos Aires (2000-2010). En Jornadas "Memoria y oportunidades en el agro argentino: burocracia, tecnología y medio ambiente (1930-2010)”. UNQUI.

Battista, S.; Feito, M. C; Cruz, A. O.; Irigoyen, M. S.; Virdó, A. N.; Aznar, M. V.; Lipera, L.; Pettinato, S.; Almonacid, C. (2014). La potencialidad del sector agrícola matancero para un proceso de desarrollo sustentable: actualidad, antecedentes y perspectivas futuras. Informe de Avance Proyecto PROINCE, UNLAM (mimeo).

Battista, S.; Feito, M. C. (2015). Territorialidad y políticas públicas. El ámbito rural de La Matanza. Dossier “Migraciones, agricultura y políticas públicas en Argentina”. Revista de Ciencias Sociales Segunda Época, UNQUI. Año7, 28.

Benencia, R.; Quaranta, G. \& Souza Casadinho, J. (comps) (2009). Cinturón Hortícola de la Ciudad de Buenos Aires. Cambios sociales y productivos. Buenos Aires: CICCUS.

Bialakowsky, A. (2006). Capitalismo y método. Alternativas de la coproducción investigativa. Laboratorio on line, año VII, 19, Otoño / Invierno 2006. Instituto de Investigaciones "Gino Germani" Facultad de Ciencias Sociales, Universidad de Buenos Aires, Argentina. 15 págs. Disponible en: http://www.sociales.uba.ar/wpcontent/uploads/9.-Capitalismo-y-m\%C3\%A9todo-N\%C2\%B026.pdf (entrada 14/08/2017)

Cáceres, D. (2015). Tecnología agropecuaria y agronegocios. La lógica subyacente del modelo tecnológico dominante. Mundo Agrario, 16(31). Recuperado a partir de http://www.mundoagrario.unlp.edu.ar/article/view/MAv16n31a08

Craviotti, C. (2008). Participación, articulación, identidad. Desafíos emergentes para la incorporación de productores familiares en el contexto de estrategias de Desarrollo Local. En P. Rodríguez Bilella y E. Tapella, Transformaciones globales y territorios. Desarrollo Rural en Argentina. Experiencias y aprendizajes. Pp 37-60. Buenos Aires: La Colmena.

Feito, M. C. (2014). Ruralidades, agricultura familiar y desarrollo. Territorio del Periurbano Norte de Buenos Aires. Buenos Aires: La Colmena.

Feito, M. C. (2016a). Si nos quitan la ilusión... no nos queda nada. Problemáticas del avance de urbanizaciones sobre producciones intensivas en el periurbano Norte de Buenos Aires. En Ruralidades, actividades económicas y mercados de trabajo en torno al Área Metropolitana de Buenos Aires. Buenos Aires: Ciccus (en prensa).

Feito, M. C. (2016b). Aportes para una ley nacional: rol de la agricultura familiar para el desarrollo rural argentino. Revista Márgenes, Espacio, Arte y Sociedad. Valparaíso: Facultad de Arquitectura de la Universidad de Valparaíso (en prensa).

FONAF, Foro de Organizaciones Nucleadas de la Agricultura Familiar (2007). Documento Base del FoNAF para implementar las políticas públicas del sector de la Agricultura Familiar. Recuperado de: http://www.fonaf.com.ar/documentos/Docmento base FoNAF.pdf

Foro de Universidades Nacionales para la Agricultura Familiar del IPAF Pampeana.

INTA (2011). Documento presentación. Buenos Aires: Ed INTA.

Instituto Nacional de Estadística y Censos INDEC (2001). Censo Nacional de Población, Hogares y Viviendas.

INDEC (2002) Censo Nacional Agropecuario.

INDEC (2010). Censo Nacional de Población, Hogares y Viviendas.

Lattuada, M., Márquez, S., y Neme, J. (2012). Desarrollo rural y política. Reflexiones sobre la experiencia argentina desde una perspectiva de gestión. Buenos Aires: Ciccus. 
Long, N. (1992). From paradigm lost to paradigm regained? The case for an actor-oriented sociology of development. En N. Long \& A. Long, Battlefields of Knowledge. The interlocking of theory and practice in social research and development. pp. 16-43. UK: Routledge.

Manzanal, M. (2002). Instituciones y gestión del desarrollo rural en la Argentina degradada: hacia la reestructuración de la Nación. Economía, Sociedad y Territorio, México. Vol. III.N 12. pp. 557-591.

Manzanal, M. (2006). Regiones, territorios e institucionalidad del Desarrollo Rural. En M. Manzanal, G. Neiman, y M. Lattuada (comps.) Desarrollo rural, organizaciones, instituciones y territorio. pp. 21-50. Buenos Aires: Ciccus

Neiman, G. (2010). Pobreza, políticas sociales y desarrollo rural. Algunas evidencias de su relación a partir de la experiencia argentina. En M. Manzanal y G. Neiman: Las agriculturas del Mercosur. Trayectorias, amenazas y desafíos. pp. 79-91. Buenos Aires: Ciccus.

Obschatko, E., Foti, M., y Román, M. (2006). Los pequeños productores en la república Argentina. Importancia de la producción agropecuaria y en el empleo en base al Censo Nacional Agropecuario 2002. Buenos Aires: SAGYP/IICA.

Oszlak, O. (2007). Formación histórica del Estado en América Latina. Elementos teórico-metodológicos para su estudio. Lecturas sobre el Estado y las políticas públicas: Retomando el debate de ayer para fortalecer el actual. Buenos Aires: Proyecto de Modernización del Estado, Jefatura de Gabinete de Ministros de la Nación.

Parés, G. (2009). Las funciones de la agricultura urbana y periurbana en La Matanza, desde la óptica de los propios agricultores, en la primera década del siglo XXI. (Tesis de maestría inédita) Universidad Nacional de San Martín / Universidad Autónoma de Madrid. Buenos Aires/Madrid.

Prividera, G., Marasas, M., Tito, G., Cittadini, R., Dumrauf, S., Alderete, F., Civeira, G., Cap, G., y Giordano, G. (2010). El desarrollo de tecnologías apropiadas para los pequeños productores de la Argentina. Marco teórico del Instituto de Investigación y Desarrollo Tecnológico para la Pequeña Agricultura Familiar en la Región Pampeana- INTA. Recuperado de: http://www.monografias.com/trabajos107/desarrollo-tecnologias-apropiadaspequenos-productores/desarrollo-tecnologias-apropiadas-pequenos-productores.shtml

Ramilo, D. (2012). Agricultura Urbana y Periurbana en el Área Metropolitana de Buenos Aires. Buenos Aires: Ed Inta.

Ratier, H. (2002). Rural, ruralidad, nueva ruralidad y contraurbanización. Un estado de la cuestión. Revista de Ciencias Humanas, 31, 1-257.

Rodríguez Bilella, P. (2004). Etnografía y política social: el caso del enfoque orientado al actor. En Actas IV Jornadas de Etnografía y Métodos Cualitativos: IDES.

Rodríguez Bilella, P. y Tapella, E. (2008). Introducción. Transformaciones Globales y Territorios: Desarrollo Rural en Argentina, Experiencias y Aprendizajes. Buenos Aires: La Colmena.

Schetjman, A. (1983). Campesinado y desarrollo rural; lineamientos de una estrategia alternativa. Investigación Económica, Vol XLII, 164. pp. 115-152.

Schiavoni, G. (2010). Describir y prescribir: la tipificación de la agricultura familiar en Argentina. En M. Manzanal y G. Neiman (comps.), Las agriculturas familiares del Mercosur. Trayectorias, amenazas y desafíos. pp. 43-61. Buenos Aires: Ciccus.

Svetlitza de Nemirovsky, A. (2002). Tendencias en la dinámica de la estructura social agraria del partido de La Matanza, 1900-2000. Documento de Trabajo $\mathrm{N}^{\circ} 88$ Universidad de Belgrano. Recuperado de: http://www.ub.edu.ar/investigaciones/dt nuevos/88 nemirovsky.pdf 
Svetlitza de Nemirovsky, A., y González, R. (1997). En torno a la historia agraria del partido de La Matanza. En Cuadernos Encuentro. Para el Aprendizaje, la Investigación y la Difusión. Universidad Nacional de La Matanza. Departamento de Humanidades y Ciencias Sociales. Para uso y divulgación de Cátedras y equipos de investigación. 9. pp. 3-20. 